Article

\title{
Intratumoral Heterogeneity of Expression of 16 miRNA in Luminal Cancer of the Mammary Gland
}

\author{
Yuliya A. Veryaskina ${ }^{1,2, *(\mathbb{D}}$, Sergei E. Titov ${ }^{2,3}{ }^{(}$, , Vlada V. Kometova ${ }^{4}$, Valerii V. Rodionov ${ }^{4}$ and \\ Igor F. Zhimulev ${ }^{2}$ \\ 1 The Federal Research Center Institute of Cytology and Genetics, \\ Siberian Branch of the Russian Academy of Sciences, 630090 Novosibirsk, Russia \\ 2 Institute of Molecular and Cellular Biology, Siberian Branch of the Russian Academy of Sciences, \\ 630090 Novosibirsk, Russia; titovse78@gmail.com (S.E.T.); zhimulev@mcb.nsc.ru (I.F.Z.) \\ 3 AO Vector-Best, 630117 Novosibirsk, Russia \\ 4 National Medical Research Center for Obstetrics, Gynecology and Perinatology named after Academician \\ V. I. Kulakov of the Ministry of Healthcare of the Russian Federation, 117997 Moscow, Russia; \\ vladastasiatema@mail.ru (V.V.K.); V_rodionov@oparina4.ru (V.V.R.) \\ * Correspondence: microrna@inbox.ru
}

Received: 3 March 2020; Accepted: 7 May 2020; Published: 11 May 2020

\begin{abstract}
The purpose of this work is to determine the intratumoral distribution of miRNA expression profiles in luminal breast cancer (BC). The study included 33 certain $\mathrm{BC}$ cases of the luminal $\mathrm{A}$ or luminal B (Her2-) subtypes. The relative expression levels of miRNA-20a; -21; -125b; -126; -200b; -181a; -205; -221; -222; -451a; -99a; -145; -200a; -214; -30a; -191; and small nuclear RNAs U6, U54, and U58 were measured by RT-qPCR in four intratumor areas in each of 33 luminal BC specimens and in surrounding normal mammary gland tissues. Comparative analysis of miRNA expression levels between normal mammary gland tissue and different intratumor areas revealed that only four miRNAs (miRNA-21, -200b, -200a, -191) appear as consistently differentiating markers. A comparative analysis of miRNA expression levels between normal mammary gland tissue and the tumor border revealed statistically significant differences for ten miRNAs; 10 miRNAs show differential expression between normal mammary gland tissue and central tumor specimens; 9 miRNAs show differential expression between normal mammary gland tissue and tumor periphery 1; 13 miRNAs show differential expression between normal mammary gland tissue and tumor periphery 2 . After comparing the tumor periphery 1 and tumor center, we found statistically significant differences in expression between five miRNAs and after comparing the tumor periphery 2 and tumor center, differences were observed for 12 miRNAs. MiRNA expression levels are subject to considerable variation, depending on the intratumor area. This may explain the inconsistency in miRNA expression estimates in BC coming from different laboratories.
\end{abstract}

Keywords: biomarker; breast cancer; miRNA; luminal breast cancer; intra-tumor heterogeneity

\section{Introduction}

Breast cancer (BC) is the most common type of cancer among women worldwide. Breast carcinomas are divided into two classes: monogenic and polygenic. Each monogenic tumor appears to contain one large clonal subpopulation with a highly stable chromosome structure. Polygenic cancers contain several clonal tumor subpopulations each [1]. Yates et al. published their results on the spatial distribution of subclones for 12 tumors, with several biopsies obtained from the tumor section surface for assessing its genetic heterogeneity. Eight of the 12 tumors demonstrated spatial heterogeneity of mutations [2]. A study looking at possible subclonality of a primary tumor demonstrated that as 
many as eight intratumor areas were required to detect $90 \%$ of genomic diversity [3]. At present, personalized anti-BC treatment to a large extent depends on tumor morphology, size, lymph-node metastasis, and the expression of such markers as the estrogen receptor (ER), the progesterone receptor (PR), human epidermal growth factor receptor 2 (HER2), and the cellular marker of proliferation Ki-67. Luminal tumors are ER-positive and represent the most prevalent BC subtype [4]. About $20 \%$ of tumors show differences in ER, PR, and HER2 at re-evaluation. The observed differences may have occurred due to both technical factors and intratumoral cellular heterogeneity [5]. Patients with a high level of intratumoral ER heterogeneity had an increased long-term risk of fatal BC [6]. Additionally, it was demonstrated that ER expression was higher at the tumor periphery than in the center [7]. HER2 is a transmembrane tyrosine kinase receptor. There was considerable variation in intratumoral heterogeneity in relation to HER2 copy number between patients because of much shorter recurrence-free survival and because there were fewer survivors in the long term [8]. Ki-67 is a nuclear proliferation marker. It has been demonstrated that it may be of prognostic value in both ER-positive and ER-negative breast carcinomas; however, expression levels of Ki-67 can be higher at the tumor periphery [9]. Apart from genetic heterogeneity, there is epigenetic heterogeneity, which also contributes to tumor intratumoral heterogeneity. MiRNAs are a class of modulators involved in cancer and may be important predictors of disease risk and progression. Quite a few miRNAs showed differential expression between different molecular subtypes of BC [10]. It has been demonstrated that the miRNA profile in breast tumor cells is not the same as in the surrounding morphologically normal tissue. Noteworthy, miRNA expression levels may not be identical in different studies-not even if the tissue type is the same [11]. Results may not be identical due to technical factors, including differences in reagents or types of analysis, sampling protocols, fixation conditions, or storage conditions for unfixed specimens. Dario de Biase et al. compared miRNA expression levels between fresh frozen and paraffin embedded glioblastoma specimens and observed a good correlation [12]. However, Vojtechova et al. showed that the overlap of differentially expressed miRNA between the fresh frozen specimens and the paraffin blocks was only about 30\% [13]. Differences in the results of different miRNA expression studies may also be due to intratumoral heterogeneity; however, little is known at the moment about the intratumoral distribution of miRNA expression levels [14].

The aim of this work is to determine the intratumoral distribution of miRNA expression profiles in luminal BC.

\section{Results}

\subsection{Choosing a Reference Gene for $q P C R$}

For miRNA quantification with RT-qPCR, a reference gene should be chosen. If the expression of the reference gene is variable within the tumor, this will have implications for the 2- $\Delta \mathrm{Ct}$ value. Therefore, the wrong choice of reference gene can be one of the reasons accounting for data inconsistency between different studies. Works exist that analyze miRNA expression levels with normalization to known reference genes (for example, to small nuclear RNA) without testing their expression for stability in the specimens of interest. However, more recent works demonstrated that the expression levels of small nuclear RNA are variable in cancer, and therefore, more and more attention is paid to the fact that not a single gene is universal for all cell types and in all experimental conditions [15]. It is also noted that a reference should have the same properties as has miRNA, because the extraction and identification efficiency for miRNAs may not be the same as that for long noncoding RNA. That is why it is assumed that the best reference gene should be in the same RNA class as that being analyzed. The reference was chosen using geNorm, an algorithm that identifies the most stable genes from among the candidates with measured expression in the specimens of interest [11]. The relative expression levels of miRNA-20a; -21; -125b; -126; -200b; -181a; -205; -221; -222; -451a; -99a; -145; -200a; -214; -30a; -191; and small nuclear RNAs U6, U54, and U58 were measured by RT-qPCR in 132 primary tumor samples and 33 normal mammary gland tissue samples. The optimum number of reference 
genes was inferred with geNorm (Table 1). This algorithm ranks the genes according to the relative stable expression value denoted by $\mathrm{M}$, where $\mathrm{M}$ is the mean pairwise variation of the expression of a gene with that of each of the other control genes. Genes with the lowest M's have the most stable expression. It is recommended to use the geometric mean of at least three most stable genes to calculate a normalization factor (NF) and the gradual inclusion of more and more control genes until the $(n+1)$ th gene's contribution to the newly calculated normalization factor $\mathrm{NF}(\mathrm{n}+1)$ is essential. To find out if more than three genes are required for normalization, we calculated the pairwise variation $(\mathrm{Vn} / \mathrm{Vn}+1)$ between two successive normalization factors $\mathrm{NFn}$ and $\mathrm{NF}(\mathrm{n}+1)$ for all the genes in question. In our opinion, the best reference in the current settings is the geometric mean of the fluorescence threshold cycles of four miRNAs: miRNA-100, miRNA-143, miRNA-126, and miRNA-125b. Although miRNA-126 and miRNA-125b expression was stable in the sample of the specimens, these miRNAs have some important functions in BC, and hence, we did not include them in the geometric mean of reference genes $[16,17]$. Further on, the geometric mean of the threshold cycles of miRNA-100 and miRNA-143 will be used as the reference.

Table 1. Assessing gene stability with geNorm to determine the optimum number of reference genes.

\begin{tabular}{ccccc}
\hline miRNA & Stability Measure (M) & V Name & NFn/NF(n+1) Variation & NFn Stability \\
\hline miRNA-143 & 1.707295837 & & 1 & \\
miRNA-100 & 1.80022993 & $\mathrm{~V} 1 / 2$ & 0.528235232 & 1.670849973 \\
miRNA-126 & 1.800986451 & $\mathrm{~V} 2 / 3$ & 0.361285079 & 1.63594884 \\
miRNA-125b & 1.811601327 & $\mathrm{~V} 3 / 4$ & 0.2027059 & 1.641631035 \\
miRNA-145 & 1.859214933 & $\mathrm{~V} / 5$ & 0.16494292 & 1.650351674 \\
miRNA-20a & 1.876414282 & $\mathrm{~V} 5 / 6$ & 0.220042327 & 1.618486051 \\
miRNA-21 & 1.933340528 & $\mathrm{~V} 6 / 7$ & 0.203421143 & 1.591868665 \\
miRNA-222 & 1.937542742 & $\mathrm{~V} 7 / 8$ & 0.147144095 & 1.58906537 \\
miRNA-181a & 1.969174988 & $\mathrm{~V} 8 / 9$ & 0.139065169 & 1.584626526 \\
miRNA-221 & 1.971881076 & $\mathrm{~V} 9 / 10$ & 0.113726124 & 1.58903484 \\
miRNA-204 & 2.00646399 & $\mathrm{~V} 10 / 11$ & 0.119749575 & 1.583226837 \\
miRNA-214 & 2.106526407 & $\mathrm{~V} 11 / 12$ & 0.109896221 & 1.587798283 \\
miRNA-30a & 2.156076925 & $\mathrm{~V} 12 / 13$ & 0.136245315 & 1.570279014 \\
U6 & 2.159702998 & $\mathrm{~V} 13 / 14$ & 0.123657069 & 1.558184739 \\
U58 & 2.256085709 & $\mathrm{~V} 14 / 15$ & 0.118561989 & 1.553964734 \\
miRNA-191 & 2.307850884 & $\mathrm{~V} 15 / 16$ & 0.117821227 & 1.548875535 \\
miRNA-99a & 2.336822525 & $\mathrm{~V} 16 / 17$ & 0.09952473 & 1.551560708 \\
miRNA-200a & 2.380047394 & $\mathrm{~V} 17 / 18$ & 0.112528959 & 1.545511535 \\
U54 & 2.391901029 & $\mathrm{~V} 18 / 19$ & 0.098671803 & 1.548786269 \\
miRNA-200b & 2.70503197 & $\mathrm{~V} 19 / 20$ & 0.116023318 & 1.552206252 \\
miRNA-205 & 2.808484382 & $\mathrm{~V} 20 / 21$ & 0.112807048 & 1.552130251 \\
miRNA-451a & 3.301276521 & $\mathrm{~V} 21 / 22$ & 0.13524655 & 1.551292987 \\
\hline
\end{tabular}

\subsection{Analysis of miRNA Expression Levels between Different Intratumor Areas and Normal Mammary Gland Tissue}

The expression levels of 16 miRNAs (miRNA-20a, -21, -125b, -126, -200b, -181a, -205, -221, -222, $-451 a,-99 a,-145,-200 a,-214,-30 a,-191)$ were measured by RT-qPCR in four different areas of each tumor (tumor center (C), opposite tumor peripheral sites (P1 and P2), and tumor border(B)) and in normal tissue (N) (Figure 1). Comparative analysis of miRNA expression levels between normal mammary gland tissue and different intratumor areas revealed that 10 miRNAs (miRNA-21, -126, -200b, -221, -222, -99a, -145, -200a, -30a, -191) show differential expression between normal mammary gland tissue and tumor border specimens $(p<0.05)$; 10 miRNAs (miRNA-21, -125b, -200b, -181a, -205, -99a, -145, -200a, -30a, -191) show differential expression between normal mammary gland tissue and central tumor specimens $(p<0.05) ; 9$ miRNAs (miRNA -21, -125b, -200b, -181a, -451a, -99a, -200a, $-30 \mathrm{a},-191)$; show differential expression between normal mammary gland tissue and tumor periphery 1 (P1) specimens $(p<0.05)$; 13 miRNAs (miRNA-20a, -21, -125b, -126, -200b, -181a, -205, -221, -222, 
$-145,-200 a,-214,-191)$ show differential expression between normal mammary gland tissue and tumor periphery 2 (P2) specimens ( $p<0.05)$; Only four miRNAs (miRNA-21, miRNA-200b, miRNA-200a, and miRNA-191) appear as consistently differentiating markers when comparing specimens taken from different intratumor areas and normal tissue $(p<0.05)$ (Table 2$)$. It should be noted that the expression level of any of these four miRNAs is lower in the normal mammary gland tissue than in the tumor specimens. The expression level of miRNA-21 increases with the distance from normal mammary gland tissue; however, neither miRNA-200b nor miRNA-200a nor miRNA-191 was shown to follow this tendency.
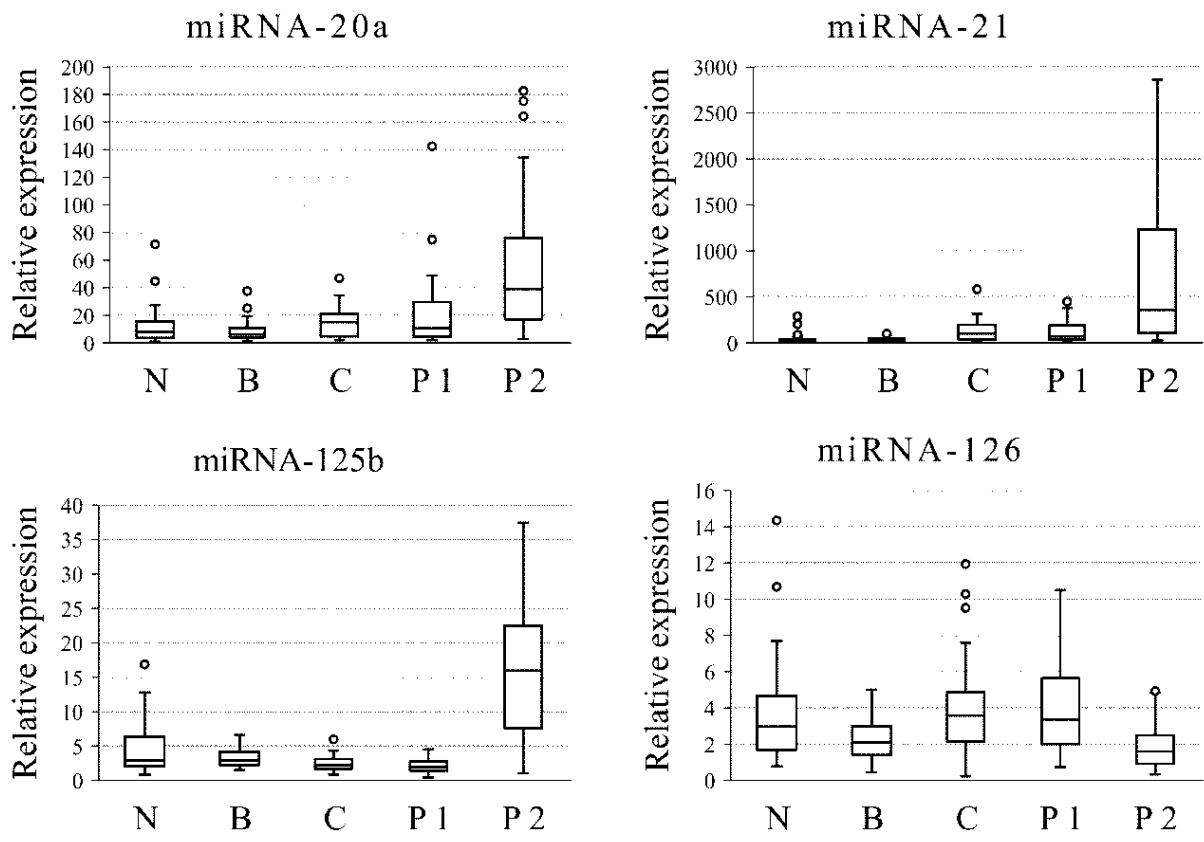

miRNA- 181 a

miRNA-200b
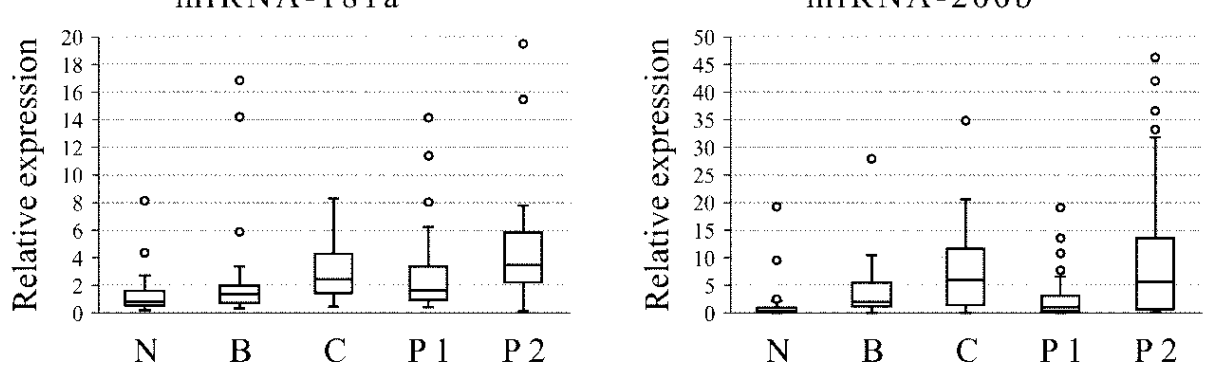

miRNA-205

miRNA-451a
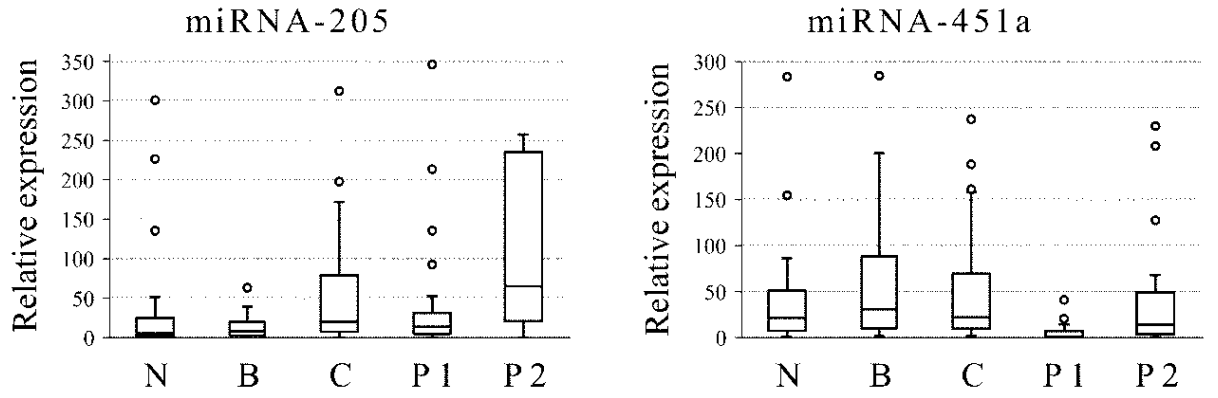

Figure 1. Cont. 
miRNA-221

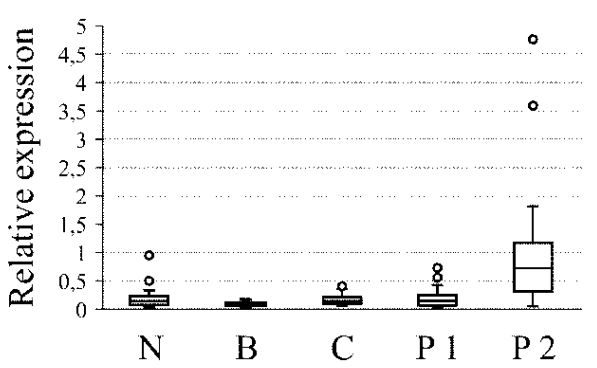

miRNA-99a

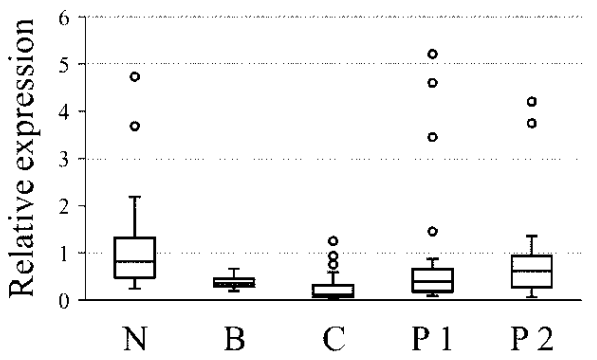

miRNA-200a

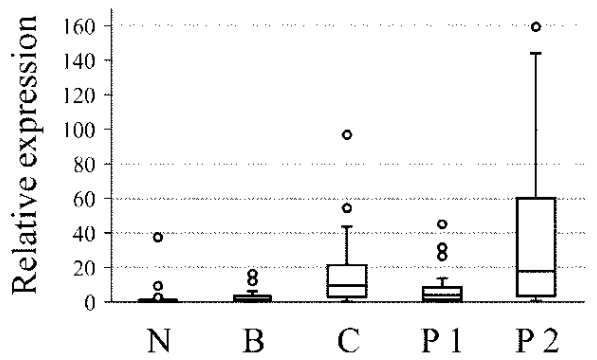

$\operatorname{miRNA}-30 a$

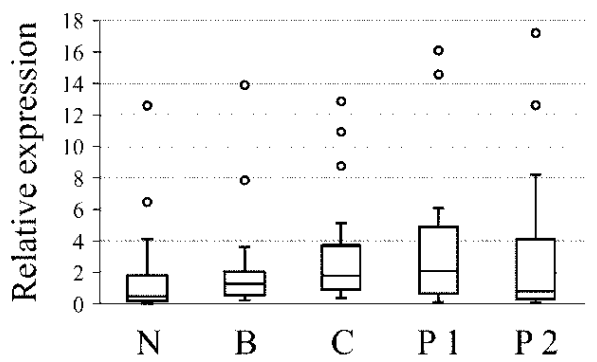

$\operatorname{miRNA}-222$

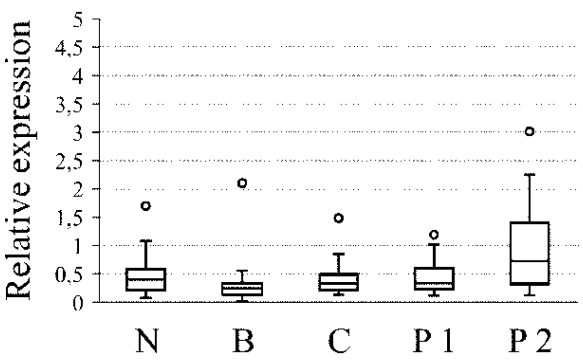

miRNA - 145

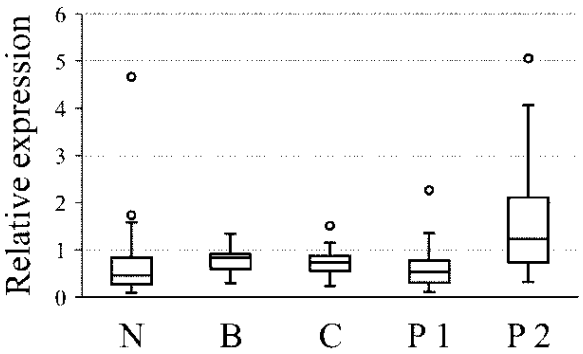

miRNA - 214

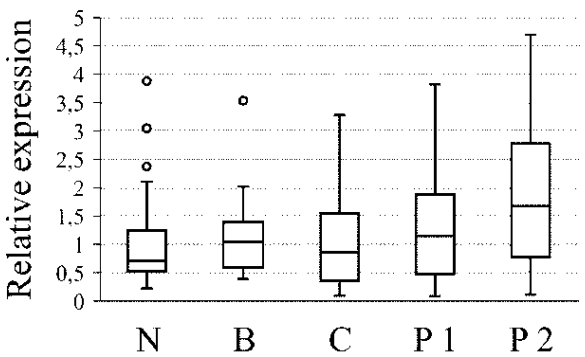

$\operatorname{miRNA}-191$

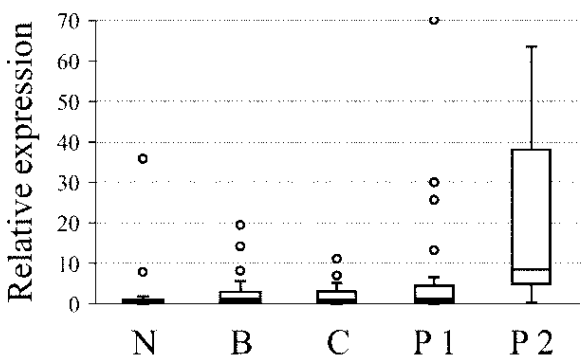

Figure 1. Comparative analysis of miRNA expression levels between specimens taken from different intratumor areas: tumor center (C), two tumor peripheries (P1 and P2), and tumor borders (B) with normal mammary gland tissue $(\mathrm{N})$. Shown are the median value, the upper and the lower quartiles, the outlier-free range, and outliers (appear as circles). 
Table 2. Significance level of differences in miRNA expression levels between normal $(n=33)$ and different intratumor areas $(n=132)$.

\begin{tabular}{|c|c|c|c|c|}
\hline miRNA & $\begin{array}{c}\text { Normal vs. Tumor } \\
\text { Border }\end{array}$ & $\begin{array}{c}\text { Normal vs. Tumor } \\
\text { Center }\end{array}$ & $\begin{array}{l}\text { Normal vs. Tumor } \\
\text { Periphery1 }\end{array}$ & $\begin{array}{l}\text { Normal vs. Tumor } \\
\text { Periphery2 }\end{array}$ \\
\hline miRNA-20a & 0.615703 & 0.062666 & 0.130845 & 0.000003 * \\
\hline miRNA-21 & 0.019136 * & 0.000001 * & 0.000006 * & 0.000000 * \\
\hline miRNA-125b & 0.561720 & 0.018225 * & 0.001328 * & 0.000002 * \\
\hline miRNA-126 & $0.017146^{*}$ & 0.683480 & 0.750119 & 0.001682 * \\
\hline miRNA-181a & 0.057594 & 0.000003 * & 0.001461 * & 0.000000 * \\
\hline miRNA-200b & 0.000029 * & 0.000000 * & 0.030546 * & 0.000004 * \\
\hline miRNA-205 & 0.911808 & 0.031572 * & 0.112178 & 0.000014 * \\
\hline miRNA-221 & 0.013697 * & 0.779285 & 0.888576 & 0.000000 * \\
\hline miRNA-222 & 0.011748 * & 0.702299 & 0.898649 & 0.001328 * \\
\hline miRNA-451a & 0.281086 & 0.18650 & 0.000000 * & 0.344958 \\
\hline miRNA-99a & 0.0000001 * & 0.000000 * & 0.000418 * & 0.081107 \\
\hline miRNA-145 & 0.002129 * & 0.020995 * & 0.637295 & 0.000008 * \\
\hline miRNA-200a & 0.000027 * & 0.000000 * & 0.000011 * & 0.000000 * \\
\hline miRNA-214 & 0.094485 & 0.959369 & 0.407011 & 0.001531 * \\
\hline miRNA-30a & 0.018452 * & 0.001095 * & 0.001934 * & 0.183532 \\
\hline miRNA-191 & 0.000425 * & 0.012171 * & 0.002541 * & 0.000000 * \\
\hline
\end{tabular}

2.3. Comparative Analysis of miRNA Expression Levels between Specimens Taken from the Tumor Border, Tumor Peripheries, and Tumor Center

Comparative analysis of miRNA expression levels between tumor border specimens and different intratumor areas revealed that 11 miRNAs (miRNA-20a, -21, -125b, -126, -200b, -181a, -205, -221, -222, -99a, -200a) show differential expression between the tumor border and the tumor center specimens $(p<0.05)$; 8 miRNAs (miRNA-20a, -21, -125b, -126, -221, -222, -451a, -145) show differential expression between the tumor border and the tumor P1 specimens ( $\mathrm{p}<0.05)$; 13 miRNAs (miRNA-20a, $-21,-125 \mathrm{~b}$, $-181 a,-205,-221,-222,-451 a,-99 a,-145,-200 a,-214,-191)$ show differential expression between the tumor border and the tumor P2 specimens $(p<0.05)$ (Table 3$)$.

Table 3. Significance level of differences in miRNA expression between specimens taken from intratumor areas and border.

\begin{tabular}{cccc}
\hline miRNA & $\begin{array}{c}\text { Tumor Border vs. } \\
\text { Tumor Center }\end{array}$ & $\begin{array}{c}\text { Tumor Border vs. } \\
\text { Tumor Periphery1 }\end{array}$ & $\begin{array}{c}\text { Tumor Border vs. } \\
\text { Tumor Periphery2 }\end{array}$ \\
\hline miRNA-20a & $0.030221^{*}$ & $0.038173^{*}$ & $0.000001^{*}$ \\
miRNA-21 & $0.000029^{*}$ & $0.000155^{*}$ & $0.000000^{*}$ \\
miRNA-125b & $0.004383^{*}$ & $0.000195^{*}$ & $0.000000^{*}$ \\
miRNA-126 & $0.005207^{*}$ & $0.008225^{*}$ & 0.226516 \\
miRNA-181a & $0.001454^{*}$ & 0.197599 & $0.000067^{*}$ \\
miRNA-200b & $0.012689^{*}$ & 0.068964 & 0.099840 \\
miRNA-205 & $0.015337^{*}$ & 0.123681 & $0.000000^{*}$ \\
miRNA-221 & $0.002448^{*}$ & $0.020571^{*}$ & $0.000000^{*}$ \\
miRNA-222 & $0.012689^{*}$ & $0.004383^{*}$ & $0.000001^{*}$ \\
miRNA-451 & 0.378257 & $0.000000^{*}$ & $0.040743^{*}$ \\
miRNA-99a & $0.000207^{*}$ & 0.911808 & $0.008915^{*}$ \\
miRNA-145 & 0.193051 & $0.005912^{*}$ & $0.000290^{*}$ \\
miRNA-200a & $0.000001^{*}$ & 0.066952 & $0.000000^{*}$ \\
miRNA-214 & 0.252791 & 0.799400 & $0.022097^{*}$ \\
miRNA-30a & 0.114274 & 0.111264 & 0.350545 \\
miRNA-191 & 0.414709 & 0.984406 & $0.000001^{*}$ \\
\hline
\end{tabular}

* statistically significant differences are in bold $(p<0.05)$. 
2.4. Comparative Analysis of miRNA Expression Levels between Specimens Taken from the Tumor Center and Tumor Peripheries

Comparative analysis of miRNA expression levels between the tumor P1 and the P2 specimens revealed that the expression levels of 13 (miRNA-20a, -21, -125b, -126, -200b, -181a, 205, 221, -222, $-451 a,-145,-200 a,-191)$ out of 16 miRNAs in question are significantly different $(p<0.05)$ (Table 4$)$. Only miRNA-126 had lower expression levels in tumor P2 than in tumor P1, while those of the other 12 miRNAs were higher in P2 than in P1. Comparative analysis of miRNA expression levels between the tumor center and two tumor peripheral sites revealed that the respective expression levels of miRNA-20a, -21, -125b, -126, -181a, -205, -221, -222, -214, -30a, and -191 are significantly different between the tumor center and the tumor P2 $(p<0.05)$ and that there is no difference in the expression of these miRNAs between the tumor center and the tumor P1. On the other hand, a significant difference has been observed in the expression levels of miRNA-200b, -451a, and -200a between the tumor center and the tumor P1 specimens $(p<0.05)$, while no significant correlation has been found between the respective expression levels of these miRNAs between the tumor center and the tumor P2. Only miRNA-99a and miRNA-145 each showed a statistically significant difference in expression level between the tumor center and either tumor peripheral site $(p<0.05)$.

Table 4. Significance level of differences in miRNA expression between specimens taken from intratumor areas.

\begin{tabular}{cccc}
\hline miRNA & $\begin{array}{c}\text { Tumor Center vs. } \\
\text { Tumor Periphery1 }\end{array}$ & $\begin{array}{c}\text { Tumor Center vs. } \\
\text { Tumor Periphery2 }\end{array}$ & $\begin{array}{c}\text { Tumor Periphery1 vs. } \\
\text { Tumor Periphery2 }\end{array}$ \\
\hline miRNA-20a & 0.918841 & $0.000077^{*}$ & $0.000946^{*}$ \\
miRNA-21 & 0.798904 & $0.000054^{*}$ & $0.000057^{*}$ \\
miRNA-125b & 0.179320 & $0.000000^{*}$ & $0.000000^{*}$ \\
miRNA-126 & 0.908738 & $0.000572 *$ & $0.000815^{*}$ \\
miRNA-181a & 0.127579 & 0.137569 & $0.006775^{*}$ \\
miRNA-200b & $0.000156^{*}$ & 0.721300 & $0.003172^{*}$ \\
miRNA-205 & 0.338451 & $0.004479 *$ & $0.000147^{*}$ \\
miRNA-221 & 0.888576 & $0.000000^{*}$ & $0.000000^{*}$ \\
miRNA-222 & 0.601311 & $0.001266^{*}$ & $0.006247^{*}$ \\
miRNA-451a & $0.000000 *$ & 0.239813 & $0.000011^{*}$ \\
miRNA-99a & $0.000357 *$ & $0.000004 *$ & 0.068390 \\
miRNA-145 & $0.035976 *$ & $0.000042 *$ & $0.000002 *$ \\
miRNA-200a & $0.007054 *$ & 0.062666 & $0.000069 *$ \\
miRNA-214 & 0.358206 & $0.006506 *$ & 0.053998 \\
miRNA-30a & 0.858470 & $0.042196 *$ & 0.106438 \\
miRNA-191 & 0.319401 & $0.000000 *$ & $0.000004 *$ \\
\hline
\end{tabular}

\section{Discussion}

We looked at the expression levels of 16 miRNAs (miRNA-20a, -21, -125b, -126, -200b, -181a, $-205,-221,-222,-451 a,-99 a,-145,-200 a,-214,-30 a,-191)$ in four intratumor areas in each of 33 luminal $\mathrm{BC}$ specimens and in surrounding normal mammary gland tissues. Our data suggest that miRNA expression levels are subject to considerable variation, depending on the intratumor area. The data obtained may be interpreted as explaining the inconsistency in miRNA expression estimates in BC coming from different laboratories.

Some works on the role of miRNA-20a in BC show its function as an oncogene with increased expression. Additionally, data published on miRNA-20a expression in different intratumor areas show that it varies across the tumor and that it is lower in tumor than in normal cells [18]. We, too, demonstrated variation in miRNA-20a expression between different intratumor areas; however, the level of expression was higher in the tumor than in the normal specimens and a statistically significant change has been observed only in the BC P2 specimens. A work by Calvano Filho et al. on the expression levels of miRNA-20a in different morphological subtypes of $\mathrm{BC}$ reports an average 
four-fold increase in miRNA-20a expression in triple negative BC as compared with the luminal subtype [19]. However, we observed a four-fold intratumor variation in miRNA-20a expression levels; thus, the choice of a sampling area within the $\mathrm{BC}$ tumor matters for the results.

It has many times been demonstrated that miRNA-125b is an oncosuppressor, and its expression is reduced in the tumor [16]. However, works exist that show miRNA-125b to be an oncogene with enhanced expression and an association with poor prognosis in BC patients and drug resistance [20]. In our study, we observed a reduction in miRNA-125b expression in the tumor P2 and the tumor center as well as its substantial increase in tumor P2 as compared with normal tissue. These data may provide evidence for cellular heterogeneity in tumors as well as for the presence of drug-resistant subclones in them.

MiRNA-205 is an oncosuppressor and the level of its expression is specific for the BC subtype, the type of the tumor-initiating cell, and the tumor stage. However, there is some controversy: one work demonstrated that miRNA-205 expression is higher in ER+/PR+/HER2+ BC than in any other BC subtypes, while another revealed a high level of miRNA-205 expression in ER-/PR-/HER2- tumors [21]. In our study, we observed a statistically significant intratumoral heterogeneity in miRNA-205 expression in luminal BC. It should be noted that there is a nearly four-fold difference in the expression level of miRNA-205 between the tumor P1 and the tumor P2 areas, which are the most spaced out intratumor locations $(p<0.05)$.

MiRNA-200a/200b are in the miRNA-200 family and act to suppress tumor development by inhibiting the epithelial-mesenchymal transition [22]. In this work, we observed elevated miRNA-200a and miRNA-200b in tumor tissue, no matter what sampling area, compared with normal tissue. It should be noted that we observed identical patterns of changes in expression for both miRNA-200a and miRNA-200b when comparing different intratumor areas.

Wang et al. found that miRNA-214 was elevated in human BC cell lines and that miRNA-214 overexpression helps avoid apoptosis. It has also been noted that miRNA-214 expression was substantially elevated in BC tissues as compared with the surrounding normal tissues [23]. However, Derfoul et al. showed that miRNA-214 inhibits BC cell proliferation and reduced miRNA-214 expression may facilitate mammary gland tumor development [24]. In our study, we observed increased miRNA-214 expression within the tumor as compared to normal tissues; however, statistically significant differences have been found only for the tumor P2 site.

MiRNA-181a is an oncogene, for it facilitates cell proliferation, its expression is elevated in $\mathrm{BC}$ and correlates with poor survival. However, evidence exists for a dual role of miRNA-181a because this miRNA was demonstrated by another work to be an oncosuppressor and a promoter of BC cell apoptosis [25]. MiRNA-21 is a marker of an aggressive BC phenotype. Evidence exists for a correlation between elevated miRNA-21, lymph-node metastasis, and the progressive disease. MiRNA-191 is an oncogene and promotes cell proliferation, migration, and chemoresistance; it is elevated in tumor tissue as compared with the normal mammary gland tissue [26]. Elevated expression of the miRNA-221/222 cluster promotes cancer cell proliferation to form an invasive phenotype and is a predictor of poor prognosis in BC patients [27]. In this work, the expression levels of the miRNA-181a, miRNA-21, and miRNA-191 oncogenes, and oncogenes-221/222 are higher within any intratumor specimen than in normal tissue; however, there are statistically significant differences in their expression levels between intratumor areas.

MiRNA-126 is antiangiogenic and its expression is reduced in BC cells [17]. MiRNA-99a is an oncosuppressor and inhibits cell proliferation and invasion, promoting apoptosis [28]. MiRNA-145 is an oncosuppressor, and its expression is reduced in BC cells [29]. We, too, observed a tendency towards a reduction in the expression levels of these miRNAs in tumor specimens as compared to normal tissue; however, we found statistically significant differences in expression not only between the tumor and the normal tissue, but also between intratumor areas. Noteworthy, we observed elevated miRNA-145 expression in the tumor P2 as compared with normal tissue. MiRNA-30a is another oncosuppressor. It inhibits invasive $\mathrm{BC}$, and its reduced expression is associated with poor prognosis 
in BC patients [30]. We observed elevated miRNA-30a in tumor tissue, no matter what sampling area, compared with normal tissue. Our results may be explained by the fact that we included in the study the luminal BC subtype, which is noted for the best prognosis.

Some miRNAs, which suppress the expression of antioncogenes play roles as oncomirs, and their expression is increased in breast cancer [31]. Other miRNAs are associated with directing their suppressor potential in breast cancer [32]. High expression of miRNA-20a significantly decreased the mRNA and protein levels of RUNX3, as well as its direct downstream targets Bim and p21. Overexpression of miRNA-20a promoted the migration and invasion of breast cancer cells in vitro [33]. MiRNA-221, miRNA-21 and miRNA-222 displayed oncogenic roles through negative regulation of PTEN [32,34]. Overexpression of miRNA-99a and decreased expression of CDC25A could suppress breast cancer cell proliferation and invasion [35]. MiRNA-30a inhibits breast cancer proliferation and metastasis by directly targeting MTDH [36]. The miR-200 family suppresses EMT by the regulation of E-cadherin [37]. Downregulation of miRNA-451a upregulated MIF expression and increased breast cancer cell growth, invasion, and tamoxifen sensitivity [38]. Other results establish the regulation of MMP-14 in cancers by miRNA-181a, and they further suggest strategies to elevate miRNA-181a to prevent cancer metastasis [39].

We demonstrated that all the miRNAs in question are differentially expressed between normal mammary gland tissue and different intratumor areas; however, only miRNA-125b, miRNA-181a, miRNA-21, miRNA-200b, miRNA-200a, and miRNA-191 are seen to be consistently differentiating markers when comparing specimens taken from all intratumor areas in question and normal tissue $(p<0.05)$ (Table 2). Thus, no matter what the sampling area is, it will not affect the measured expression of these miRNAs. Noteworthy, a comparative analysis of miRNA expression levels between normal mammary gland tissue and their border revealed statistically significant differences for 10 miRNAs, suggesting possible distinctions in the morphological and genetic features of the tumor border.

After comparing the tumor P1 and tumor center specimens, we found statistically significant differences in expression between five miRNAs, and after comparing the tumor P2 and tumor center specimens, differences were observed for 12 miRNAs. At this point, we cannot say what exactly makes the tumor P2 specimens so different from the tumor P1 ones that the differences in miRNA expression are that great. Again, P1 and P2 are the most spaced out intratumor locations. It is possible that this special configuration of the specimens can explain the highest heterogeneity in expression levels among all miRNAs being discussed.

\section{Materials and Methods}

\subsection{Materials}

The study included 33 certain BC cases of the luminal A or luminal 2 (Her2-) subtypes. The patients had received no adjuvant therapy before surgery. The study material was in the form of tumor and adjacent morphologically unchanged tissue imprinted on the slides. For each case, tumor imprints were taken from four different tumor areas (tumor center, opposite tumor peripheral sites, and tumor border) and a normal tissue imprint from adjacent morphologically unchanged tissue. Permission for this study was granted by the Biomedical Research Ethics Committee in the Kulakov Federal Research Center for Obstetrics, Gynecology, and Perinatology of the Ministry of Health of the Russian Federation. The research was carried out under the law of the Russian Federation, the ethical norms and principles set out in the Declaration of Helsinki (1964), with all additions and amendments in relation to scientific research using human biomaterial, and according to the International Ethical Guidelines for Biomedical Research Involving Human Subjects published by the Council for International Organizations of Medical Sciences (CIOMS). All the patients' source data were anonymized in accordance with the requirements set out in clause 3 of Article 6 of the current Russian Federal Law on Personal Data (No. 152-FZ). Written informed consent was obtained from each patient. Biomaterial was sampled according to the Standard Operating Procedure (SOP) protocol developed by the Kulakov Federal 
Research Center for Obstetrics, Gynecology, and Perinatology of the Ministry of Health of the Russian Federation in accord with Order of the Ministry of Health of the Russian Federation No. 179нон March 24, 2016 "On the Rules for Pathoanatomical Research" and the clinical recommendations laid down in the "Standard Technological Procedures in Pathoanatomical Research" by the Russian Society of Pathologists.

\subsection{RNA Extraction}

Nucleic acids were extracted from specimens using the RealBest Extraction 100 Reagent Kit (Vector-Best, Novosibirsk, Russia). Mammary gland tissue was washed off the slide with $600 \mu \mathrm{L}$ of guanidine lysis buffer. The suspension was mixed vigorously in a TS-20 thermo-shaker (Biosan, Riga, Latvia) for $15 \mathrm{~min}$ at $65^{\circ} \mathrm{C}$. The solution was then centrifuged for $2 \mathrm{~min}$ at 10,000 rpm (Eppendorf MiniSpin, Humburg, Germany), the supernatant was transferred to fresh tubes and supplemented with an equal volume of isopropanol and $10 \mu \mathrm{L}$ of magnetic bead suspension. That was mixed and allowed to stay for $4 \mathrm{~min}$ at room temperature. At the next extraction stage, the solution was centrifuged for $10 \mathrm{~min}$ at 13,000 rpm (Eppendorf MiniSpin, Humburg, Germany), the supernatant was removed, the pellet was washed with $500 \mu \mathrm{L}$ of $70 \%$ ethanol and then with $300 \mu \mathrm{L}$ of acetone. The precipitate was dried and dissolved in $200 \mu \mathrm{L}$ of eluting solution.

\subsection{Reverse Transcription}

The reverse transcription reaction for cDNA was carried out in a volume of $30 \mu \mathrm{L}$. Ready-for-use reactions RealBest RT Master Mix (Vector-Best, Novosibirsk, Russia) were utilized. The reverse transcription reaction contained $3 \mu \mathrm{L}$ of extracted RNA, $16.2 \mu \mathrm{L}$ of $40 \%$ threhalose solution, $3 \mu \mathrm{L}$ of $10 \times$ reverse transcription buffer, $3 \mu \mathrm{L}$ of $4 \mathrm{mM}$ deoxynucleotide triphosphate solution, $3 \mu \mathrm{L}$ of $10 \%$ bovine serum albumin solution (BSA) solution, $0.32 \mu \mathrm{L}$ of reverse transcriptase (Vector-Best, Novosibirsk, Russia), and $1.5 \mu \mathrm{L}$ of $10 \mu \mathrm{M}$ primer solution for reverse transcription. All oligonucleotides were synthesized by Vector-Best (Novosibirsk, Russia). Oligonucleotides were selected using the PrimerQuest online service (http://eu.idtdna.com/). The sequences of primers and fluorescently labeled probes are available in Supplementary Table S1. Three microliters of the reaction mixture containing cDNA was used immediately as a template for a real-time PCR on a CFX96 system (Bio-Rad, California, USA).

\subsection{Real-time PCR}

MiRNA expression levels were measured by real-time PCR on a CFX96 amplifier (Bio-Rad Laboratories, California, USA). The reaction was carried out in a volume of $30 \mu \mathrm{L}$ containing $3 \mu \mathrm{L}$ of cDNA, $14 \mu \mathrm{L}$ of $\mathrm{H} 2 \mathrm{O}, 3 \mu \mathrm{L}$ of $10 \times$ PCR buffer (Vector-Best), $3 \mu \mathrm{L}$ of $4 \mathrm{mM}$ deoxynucleotide triphosphate solution, $3 \mu \mathrm{L}$ 10\% BSA solution, $1 \mu \mathrm{L}$ of Taq polymerase (Vector-Best, Novosibirsk, Russia) along with monoclonal antibodies to its active center (Clontech, California, USA), $3 \mu \mathrm{L}$ of a mix of forward and reverse primer $(5 \mu \mathrm{M})$ and probe $(2.5 \mu \mathrm{M})$. The primers and the probes are Vector-Best (Novosibirsk, Russia) developments, the efficiency of the PCR being $85 \%-100 \%$. Analysis of the threshold cycles generated by the qPCR was performed using the $2-\Delta C t$ method. Statistical processing of data was carried out using STATISTICA v12.0 (StatSoft Inc., OK, USA). Two independent samples were compared using the Mann-Whitney $U$ test.

\subsection{GeNorm Algorithm}

The geometric mean of the quantification cycles of two miRNAs (NF2) was calculated by Formula (1):

$$
C_{q}(N F 2)=\sqrt{C_{q 1} * C_{q 2}}
$$

The most stable reference genes were selected using geNorm algorithm: let there be data on the expression $\mathrm{n}$ of various miRNAs in m samples; for each pair of miRNAs, the vector $A_{j k}$ was calculated, 
the components of which are computed as the logarithm to the base two of the ratio between the level of miRNA expression in a single sample (Formula (2)):

$$
A_{j k}=\left\{\log _{2}\left(\frac{a_{1 j}}{a_{1 k}}\right), \log _{2}\left(\frac{a_{2 j}}{a_{2 k}}\right), \ldots, \log _{2}\left(\frac{a_{m j}}{a_{m k}}\right)\right\}, \forall_{j, k} \in[1, n], k \neq j
$$

The pairwise variation $V_{j k}$ of miRNA defined as standard derivation of $A_{j k}$ elements (Formula (3)):

$$
V_{j k}=\sum_{k=1}^{n} \operatorname{st} \cdot \operatorname{dev}\left(A_{j k}\right), k \neq j
$$

The expression stability of miRNA ${ }_{\mathrm{j}}\left(M_{j}\right)$ is the arithmetic mean of all pairwise variations $V_{j k}$ (Formula (4)).

$$
M_{j}=\frac{\sum_{k=1}^{n} V_{j k}}{n-1}, k \neq j
$$

Supplementary Materials: The following are available online at http://www.mdpi.com/2311-553X/6/2/16/s1, Table S1: The oligonucleotide sequences used in the study.

Author Contributions: Y.A.V. conceived the study. V.V.K. and V.V.R. collected the materials. Y.A.V. and S.E.T. analyzed the data and performed the experiments. V.V.R. and I.F.Z. provided the resources and supervised the study. All authors read and approved the final manuscript.

Funding: This work was supported by Institute Molecular and Cellular Biology of the Siberian Branch of the Russian Academy of Sciences project (0310-2019-0003) and the grant from the Russian Science Foundation (20-14-00074).

Acknowledgments: The authors would like to express their sincere gratitude to the reviewers for their constructive comments.

Conflicts of Interest: All the authors declare that they have no conflicts of interest.

\section{References}

1. Navin, N.; Krasnitz, A.; Rodgers, L.; Cook, K.; Meth, J.; Kendall, J.; Riggs, M.; Eberling, Y.; Troge, J.; Grubor, V.; et al. Inferring tumor progression from genomic heterogeneity. Genome Res. 2010, 2, 68-80. [CrossRef] [PubMed]

2. Yates, L.R.; Gerstung, M.; Knappskog, S.; Desmedt, C.; Gundem, G.; van Loo, P.; Aas, T.; Alexandrov, L.B.; Larsimont, D.; Davies, H.; et al. Subclonal diversification of primary breast cancer revealed by multiregion sequencing. Nat. Med. 2015, 2, 751-759. [CrossRef] [PubMed]

3. Ellsworth, R.E.; Toro, A.L.; Blackburn, H.L.; Decewicz, A.; Deyarmin, B.; Mamula, K.A.; Costantino, N.S.; Hooke, J.A.; Shriver, C.D.; Ellsworth, D.L. Molecular Heterogeneity in Primary Breast Carcinomas and Axillary Lymph Node Metastases Assessed by Genomic Fingerprinting Analysis. Cancer Growth Metastasis 2015, 8, 15-24. [CrossRef] [PubMed]

4. Dai, X.; Li, T.; Bai, Z.; Yang, Y.; Liu, X.; Zhan, J.; Shi, B. Breast cancer intrinsic subtype classification, clinical use and future trends. Am. J. Cancer Res. 2015, 2, 2929-2943.

5. Allott, E.H.; Geradts, J.; Sun, X.; Cohen, S.M.; Zirpoli, G.R.; Khoury, T.; Bshara, W.; Chen, M.; Sherman, M.E.; Palmer, J.R.; et al. Intratumoral heterogeneity as a source of discordance in breast cancer biomarker classification. Breast Cancer Res. 2016, 2, 68. [CrossRef] [PubMed]

6. Lindström, L.S.; Yau, C.; Czene, K.; Thompson, C.K.; Hoadley, K.A.; Van't Veer, L.J.; Balassanian, R.; Bishop, J.W.; Carpenter, P.M.; Chen, Y.Y.; et al. Intratumor, Heterogeneity of the Estrogen Receptor and the Long-term Risk of Fatal Breast Cancer. J. Natl. Cancer Inst. 2018, 2, 726-733. [CrossRef] [PubMed]

7. Douglas-Jones, A.G.; Collett, N.; Morgan, J.M.; Jasani, B. Comparison of core oestrogen receptor (ER) assay with excised tumour: Intratumoral distribution of ER in breast carcinoma. J. Clin. Pathol. 2001, 2, 951-955. [CrossRef]

8. Rye, I.H.; Trinh, A.; Saetersdal, A.B.; Nebdal, D.J.H.; Lingjærde, O.C.; Almendro, V.; Polyak, K.; Børresen-Dale, A.L.; Helland, Å.; Markowetz, F.; et al. Intratumor heterogeneity defines treatment-resistant HER2+ breast tumors. Mol. Oncol. 2018, 2, 1838-1855. [CrossRef]

9. Turashvili, G.; Brogi, E. Tumor Heterogeneity in Breast Cancer. Front. Med. 2017, 4, 227. [CrossRef] 
10. Blenkiron, C.; Goldstein, L.D.; Thorne, N.P.; Spiteri, I.; Chin, S.F.; Dunning, M.J.; Barbosa-Morais, N.L.; Teschendorff, A.E.; Green, A.R.; Ellis, I.O.; et al. MiRNA expression profiling of human breast cancer identifies new markers of tumor subtype. Genome Biol. 2007, 2, R214. [CrossRef]

11. Vandesompele, J.; De Preter, K.; Pattyn, F.; Poppe, B.; van Roy, N.; de Paepe, A.; Speleman, F. Accurate normalization of real-time quantitative RT-PCR data by geometric averaging of multiple internal control genes. Genome Biol. 2002, 3. [CrossRef] [PubMed]

12. De Biase, D.; Visani, M.; Morandi, L.; Marucci, G.; Taccioli, C.; Cerasoli, S.; Baruzzi, A.; Pession, A.; PERNO Study Group. miRNAs expression analysis in paired fresh/frozen and dissected formalin fixed and paraffin embedded glioblastoma using real-time pCR. PLoS ONE 2012, 2, e35596. [CrossRef] [PubMed]

13. Vojtechova, Z.; Zavadil, J.; Klozar, J.; Grega, M.; Tachezy, R. Comparison of the miRNA expression profiles in fresh frozen and formalin-fixed paraffin-embedded tonsillar tumors. PLoS ONE 2017, 2, e0179645. [CrossRef] [PubMed]

14. Raychaudhuri, M.; Schuster, T.; Buchner, T.; Malinowsky, K.; Bronger, H.; Schwarz-Boeger, U.; Höfler, H.; Avril, S. Intratumoral heterogeneity of miRNA expression in breast. Cancer J. Mol. Diagn. 2012, 14, 376-384. [CrossRef]

15. Lou, G.; Ma, N.; Xu, Y.; Jiang, L.; Yang, J.; Wang, C.; Jiao, Y.; Gao, X. Differential distribution of U6 (RNU6-1) expression in human carcinoma tissues demonstrates the requirement for caution in the internal control gene selection for miRNA quantification. Int. J. Mol. Med. 2015, 36, 1400-1408. [CrossRef]

16. Feliciano, A.; Castellvi, J.; Artero-Castro, A.; Leal, J.A.; Romagosa, C.; Hernández-Losa, J.; Peg, V.; Fabra, A.; Vidal, F.; Kondoh, H.; et al. miR-125b acts as a tumor suppressor in breast tumorigenesis via its novel direct targets ENPEP CK2- $\alpha$ CCNJ and MEGF9. PLOS ONE 2013, 2, e76247. [CrossRef]

17. Wang, C.Z.; Yuan, P.; Li, Y. MiR-126 regulated breast cancer cell invasion by targeting ADAM9. Int. J. Clin. Exp. Pathol. 2015, 2, 6547-6553.

18. Li, J.Y.; Zhang, Y.; Zhang, W.H.; Jia, S.; Kang, Y.; Zhu, X.Y. Differential distribution of miR-20a and miR-20b may underly metastatic heterogeneity of breast cancers. Asian Pac. J. Cancer Prev. 2012, 2, 1901-1906. [CrossRef]

19. Calvano Filho, C.M.; Calvano-Mendes, D.C.; Carvalho, K.C.; Maciel, G.A.; Ricci, M.D.; Torres, A.P.; Filassi, J.R.; Baracat, E.C. Triple-negative and luminal, A.; breast tumors: Differential expression of miR-18a-5p, miR-17-5p, and miR-20a-5p. Tumour Biol. 2014, 35, 7733-7741. [CrossRef]

20. Luo, Y.; Wang, X.; Niu, W.; Wang, H.; Wen, Q.; Fan, S.; Zhao, R.; Li, Z.; Xiong, W.; Peng, S.; et al. Elevated miRNA-125b levels predict a worse prognosis in HER2-positive breast cancer patients. Oncol. Lett. 2016, 2, 867-874.

21. Greene, S.B.; Herschkowitz, J.I.; Rosen, J.M. The ups and downs of miR-205: Identifying the roles of miR-205 in mammary gland development and breast cancer. RNA Biol. 2010, 2, 300-304. [CrossRef] [PubMed]

22. Park, S.M.; Gaur, A.B.; Lengyel, E.; Peter, M.E. The miR-200 family determines the epithelial phenotype of cancer cells by targeting the, E.;-cadherin repressors ZEB1 and ZEB2. Genes Dev. 2008, 2, 894-907. [CrossRef] [PubMed]

23. Wang, F.; Li, L.; Chen, Z.; Zhu, M.; Gu, Y. MiRNA-214 acts as a potential oncogene in breast cancer by targeting the PTEN-PI3K/Akt signaling pathway. Int. J. Mol. Med. 2016, 37, 1421-1428. [CrossRef] [PubMed]

24. Derfoul, A.; Juan, A.H.; Difilippantonio, M.J.; Palanisamy, N.; Ried, T.; Sartorelli, V. Decreased miRNA-214 levels in breast cancer cells coincides with increased cell proliferation, invasion and accumulation of the Polycomb Ezh2 methyltransferase. Carcinogenesis 2011, 2, 1607-1614. [CrossRef] [PubMed]

25. Yang, C.; Tabatabaei, S.N.; Ruan, X.; Hardy, P. The dual regulatory role of mir-181a in breast cancer. Cell Physiol. Biochem. 2017, 44, 843-856. [CrossRef]

26. Mar-Aguilar, F.; Luna-Aguirre, C.M.; Moreno-Rocha, J.C.; Araiza-Chavez, J.; Trevino, V.; Rodriguez-Padilla, C.; Resendez-Perez, D. Differential expression of miR-21, miR-125b and miR-191 in breast cancer tissue. Asia-Pacific. J. Clin. Oncol. 2013, 9, 53-59. [CrossRef]

27. Cheng, C.W.; Yu, J.C.; Hsieh, Y.H.; Liao, W.L.; Shieh, J.C.; Yao, C.C.; Lee, H.J.; Chen, P.M.; Wu, P.E.; Shen, C.Y. Increased cellular levels of miRNA-9 and miRNA-221 correlate with cancer stemness and predict poor outcome in human breast. Cancer Cell Physiol. Biochem. 2018, 48, 2205-2218. [CrossRef]

28. Wang, X.; Li, Y.; Qi, W.; Zhang, N.; Sun, M.; Huo, Q.; Cai, C.; Lv, S.; Yang, Q. MiRNA-99a inhibits tumor aggressive phenotypes through regulating HOXA1 in breast cancer cells. Oncotarget 2015, 2, 32737-32747. 
29. Zhao, H.; Kang, X.; Xia, X.; Wo, L.; Gu, X.; Hu, Y.; Xie, X.; Chang, H.; Lou, L.; Shen, X. miR-145 suppresses breast cancer cell migration by targeting FSCN-1 and inhibiting epithelial-mesenchymal transition. Am. J. Transl. Res. 2016, 2, 3106-3114.

30. Cheng, C.W.; Wang, H.W.; Chang, C.W.; Chu, H.W.; Chen, C.Y.; Yu, J.C. MiRNA-30a inhibits cell migration and invasion by downregulating vimentin expression and is a potential prognostic marker in breast cancer. Breast Cancer Res. Treat. 2012, 2, 1081-1093. [CrossRef]

31. Loh, H.Y.; Norman, B.P.; Lai, K.S.; Rahman, N.M.A.N.A.; Alitheen, N.B.M.; Osman, M.A. The Regulatory Role of MicroRNAs in Breast. Cancer Int. J. Mol. Sci. 2019, 20, 4940. [CrossRef]

32. Prabhu, K.S.; Raza, A.; Karedath, T.; Raza, S.S.; Fathima, H.; Ahmed, E.I.; Kuttikrishnan, S.; Therachiyil, L.; Kulinski, M.; Dermime, S.; et al. Non-Coding RNAs as Regulators and Markers for Targeting of Breast Cancer and Cancer Stem Cells. Cancers 2020, 12, 351. [CrossRef]

33. Bai, X.; Han, G.; Liu, Y.; Jiang, H.; He, Q. MiRNA-20a-5p promotes the growth of triple-negative breast cancer cells through targeting RUNX3. Biomed. Pharmacother. 2018, 103, 1482-1489. [CrossRef]

34. Wang, N.; Zhu, C.; Xu, Y.; Qian, W.; Zheng, M. Negative, Regulation of PTEN by MicroRNA-221 and Its Association with Drug Resistance and Cellular Senescence in Lung, Cancer Cells. Biomed. Res. Int. 2018, 2018, 7908950. [CrossRef]

35. Qin, H.; Liu, W. MicroRNA-99a-5p suppresses breast cancer progression and cell-cycle pathway through downregulating CDC25A. J. Cell Physiol. 2019, 2, 3526-3537. [CrossRef]

36. Zhang, N.; Wang, X.; Huo, Q.; Sun, M.; Cai, C.; Liu, Z.; Hu, G.; Yang, Q. MicroRNA-30a suppresses breast tumor growth and metastasis by targeting metadherin. Oncogene 2014, 2, 3119-3128. [CrossRef]

37. Korpal, M.; Lee, E.S.; Hu, G.; Kang, Y. The miR-200 family inhibits epithelial-mesenchymal transition and cancer cell migration by direct targeting of E-cadherin transcriptional repressors ZEB1 and ZEB2. J. Biol. Chem. 2008, 283, 14910-14914. [CrossRef]

38. Liu, Z.; Miao, T.; Feng, T.; Jiang, Z.; Li, M.; Zhou, L.; Li, H. miR-451a Inhibited Cell Proliferation and Enhanced Tamoxifen Sensitive in Breast Cancer via Macrophage Migration Inhibitory Factor. Biomed. Res. Int. 2015, 2015, 207684. [CrossRef]

39. Li, Y.; Kuscu, C.; Banach, A.; Zhang, Q.; Pulkoski-Gross, A.; Kim, D.; Liu, J.; Roth, E.; Li, E.; Shroyer, K.R.; et al. miR-181a-5p Inhibits Cancer Cell Migration and Angiogenesis via Downregulation of Matrix Metalloproteinase-14. Cancer Res. 2015, 2, 2674-2685. [CrossRef] 